Bull. Fac .Agric., Cairo Univ. 64:116-128 (2013).

\title{
EFFECT OF SOME GROWTH REGULATORS UNDER DIFFERENT PLANTING DATES ON GROWTH AND YIELD OF COTTON
}

\author{
(Received:18 .4 .2013)
}

\author{
By \\ S. E.D. Elayan, A. M. Abdallah, M .A. Elsayed * and R. M. Abdel-Twab* \\ Department of Agronomy, Faculty of Agriculture, Cairo University, Egypt \\ * Department of Spinning, Cotton Research Institute, Agriculture Research Center, Giza, Egypt
}

\begin{abstract}
This study was carried out at the Experiment and Research Station, Faculty of Agriculture, Cairo University, Giza in two growing seasons (2010 and 2011) to study the effect of three sowing dates; (25 March, 25 April, and 25 May) and some growth regulators i.e.,(Indole acetic acid (IAA) at the rates of 50, 100 and $150 \mathrm{ppm}$, Naphthalene acetic acid (NAA) at the rates of 10, 20 and $30 \mathrm{ppm}$ and Mepiquate chloried (Pix) 50, 100 and I50 ppm) on Egyptian cotton cultivar (Giza 92). A split-plot design with three replicates was used, sowing dates were allocated in the main plot while growth regulators in the sub plot. Obtained results could be summarized as follows:-

Sowing date had significant effect on growth characters, yield, yield components and technological properties. Whereas, delayed sowing date tended to increase significantly the plant height, no. of days to $1^{\text {st }}$ bud, $1^{\text {st }}$ flower and $1^{\text {st }}$ open boll. Planting cotton in 25 March was superior in the no. of sympodial branches / plant, seed index, earliness percentage, seed cotton yield /feddan and fiber properties such as fiber length, uniformity ratio, fiber elongation, fiber strength, micronaire reading, brightness and yellowness.

Growth regulators significantly affected all growth and yield characters under study. Use of $50 \mathrm{ppm}$ PIX was superior in the no. of days to $1^{\text {st }}$ bud, $1^{\text {st }}$ flower and $1^{\text {st }}$ open boll, seed index, earliness percentage and seed cotton yield /feddan, while $30 \mathrm{ppm}$ NAA was superior in plant height. Fiber properties were not significantly affected by growth regulators in all characters under study in both seasons.

The interaction between sowing dates and growth regulators significantly affected in most characters under study whereas, the use of 50 ppm PIX and planting in 25 March or 25 April was superior in the no. of sympodial branches / plant, no. of open bolls / plant, seed index, earliness percentage and seed cotton yield /feddan in the first season, while in the second one the use of 50 or $100 \mathrm{ppm}$ PIX with sowing in 25 March gave the best reading in yield and yield components.
\end{abstract}

Key words: cotton plant, growth and yield, growth regulators, planting dates.

\section{INTRODUCTION}

Cotton (Gossypium barbadense, L.) is one of the most important fiber crops all over the world. In Egypt its importance is derived from being one of the main sources of foreign currency as well as the principle raw material for the national textile industry and an important source of edible oil. Sowing date is considered the most important factor among the different critical factors which influence growth and yield of cotton. These effects depend mainly on the weather conditions prevailing, such as temperature, light, relative humidity and other climatic factors. Early sowing has a favorable effect on the yield of cotton compared with the late sowing, having marked effects on growth and development of cotton plant.

Emara et al. (2006) found that, number of open bolls per plant, yield / feddan and lint percentage were increased in favour of early sowing, while late sowing increased boll weight and seed index. Zakaria et al. (2006) reported the effect of mepiquate chloride at 70 and 95 days after planting and observed increased number of opened bolls per plant, boll weight, seed index, lint yield per plant and lint yield per hectare. Hakoomat et al. (2009) used three sowing dates of May 10, June 1 and June 20 during two consecutive crop seasons. Observations were recorded for yield attribute, such as the number of 
sympodial branches/plant, the number of bolls $/ \mathrm{m}^{2}$, boll weight, seed index and yield of seed cotton. Hayatullah et al. (2011) found that, late sowing consumed more the number of heat units and late planting had the highest the number of heat units and resulted in increasing plant height. Early sowing, (25 April) significantly decreased plant height, increase in earliness percentage and the number of days from sowing to the $1^{\text {st. }}$ flower and the $1^{\text {st. }}$ open boll was decreased by delaying sowing dates until 25 May as a result of increasing air temperatures. Zhiguo et al. (2011) reported that, early sowing dates significantly increased fiber strength. Wenqing et al. (2012) revealed that planting cotton early on (27 April) had significantly increased the number of sympodia on the main-stem, the number of open bolls per plant, boll weight, seed index,, lint percentage, earliness percentage and seed cotton yield, per plant and feddan, as compared with late sowing on (25 May).

Growth regulators are one of the most important factors that affect crop growth and yield. It is well known that vegetative characters, yield and its components and earliness measurements of cotton are governed by many environmental factors such as planting date which ranks high among the important factors affecting cotton production, while growth regulators application to cotton plants can modify some developmental activities such as boll setting in relation to yield and yield components.

Stephen et al. (2007) found that the application of mepiquate chloride (PIX) increased the yield and its components, i.e, the number of open bolls per plant, boll weight and yield per plant. Emara $e t$ al. (2009) reported that, the final plant height at harvest, length of internodes, days to appearance of the first flower and first boll were decreased significantly in the case of plant treatment by Mepiquate chloride. While,the number of open bolls per plant, seed cotton yield per plant and per feddan, and micronaire reading were increased significantly by applying Mepiquate chloride, number of fruiting branches and seed index were increased by mepiquate chloride in one season only. The number of nodes per plant, boll weight, number of plants at harvest, lint percentage, uniformity ratio, fiber strength, fiber elongation percentage, reflectance and yellowness were insignificantly affected. Shahzad et al. (2012) indicated that PIX and Naphthalene acetic acid significantly affected the volume of bolls and yield of cotton whereas, the use of 50 ppm PIX gave the highest number of open bolls/ plant, boll weight, seed index and earliness percentage.

The main objective of this study was to investigate the effect of different sowing dates and some growth regulators on growth and earliness traits, yield and fiber properties of Giza 92 cotton cultivar.

\section{MATERIALS AND METHODS}

Two field experiments were carried out at the Agriculture Experiment and Research Station, Faculty of Agriculture, Cairo University, Giza for two seasons (2010 and 2011), to study the effect of three sowing dates (25 March, 25 April, and 25 May) and three growth regulators; Indole acetic acid (IAA) at the rates of 50, 100 and I50 ppm, Naphthalene acetic acid (NAA) at the rates of 10, 20 and $30 \mathrm{ppm}$ and Mepiquate chloride (PIX) at the rates 50, 100 and $\mathrm{I} 50 \mathrm{ppm}$ on growth, yield and fiber quality. The spraying time was at pine head square, at the beginning of flowering and after flowering by two weeks on the Egyptian cotton variety (Giza 92). The experimental design was a split-plot with three replicates with sowing date in the main plots and growth regulators in the sub plots. Sub plot consisted of 6 ridges $60 \mathrm{~cm}$ apart and 4 meters long $\left(14.4 \mathrm{~m}^{2}\right)$. All cultural practices were done as recommended for the region. Nitrogen $(60 \mathrm{~kg} \mathrm{~N} /$ fed $)$ as ammonium nitrate $(33.5 \% \mathrm{~N})$ and potassium $\left(48 \mathrm{~kg} \mathrm{~K}_{2} \mathrm{O} / \mathrm{fed}\right)$ as potassium sulphate $\left(48 \% \quad \mathrm{~K}_{2} \mathrm{O}\right)$ were side dressed before the first and the second irrigations. Phosphorus (30 kg $\mathrm{P}_{2} \mathrm{O}_{5} / \mathrm{fed}$ ) as super phosphate $\left(\begin{array}{lll}15.5 \% & \mathrm{P}_{2} \mathrm{O}_{5}\end{array}\right)$ was broadcasted during seedbed preparation. The preceding crop was berseem in both seasons. Analysis of variance of different traits was preceded and the treatment means were compared by LSD as outlined by Sndecor and Cochran (1981). Ten plants were taken at random from each plot at 105 days from sowing to determine growth attributes and some yield components while seed cotton yield / feddan was determined on the basis of the yield per plot.

\subsection{Recorded data: \\ 2.1.1.Growth attributes}

2.1.1.1.Plant height $(\mathrm{cm})$.

2.1.1.2. Phenological stages, the number of days to the $1^{\text {st }}$ bud, $1^{\text {st }}$ flower and $1^{\text {st }}$ open boll.

\subsubsection{Yield and yield components}

2.1.2. 1- Number of sympodial branches/plant.

2.1.2. 2- Number of open bolls / plant.

2.1.2. 3- Boll weight (gm): the average of 50 bolls weight picked at random from each plot.

2.1.2. 4- Seed index: as the weight of 100 seeds. 
2.1.2. 5. Earliness percentage: percentage of seed cotton yield at the first pick to the total seed cotton yield / plot.

$$
\begin{aligned}
& \text { Seed cotton yield at first pick }(\mathrm{kg}) \\
& \text { Earliness \% = - } \\
& \text { Total seed cotton yield }(\mathrm{kg})
\end{aligned}
$$

2.1.2. 6. Seed cotton yield / feddan: Determined from the three central ridges of each plot in kentar $/$ fed. $($ Kentar $=157.50 \mathrm{~kg}$. $)$.

\subsubsection{Fiber properties}

The following fiber properties were measured using Instrument (HVI).High volume fiber test system according to (A.S.T.M: D 46050 - 1998).

2.1.3. 1. Fiber length at upper half mean (U.H.M) $\mathrm{mm}$.

2.1.3. 2. Fiber uniformity index (U.I).

2.1.3. 3. Fiber strength in gram / tex.

2.1.3. 4. Fiber elongation $\%$.

2.1.3. 5. Fiber fineness. (micronair).

2.1.3. 6. Color attributes values.

All fiber tests were performed at the laboratories of the Cotton Research Institute, Agricultural Research Center, under constant conditions of temperature $\left(70^{\circ} \mathrm{f} \pm 2\right)$ and relative humidity $(65 \% \pm 2 \%)$.

\section{RESULTS AND DISCUSSION \\ 3.1.Effect of sowing date and growth regulators on}

\subsubsection{Growth attributes \\ 3.1.1.1. Plant height}

Data in Tables (1, 2 and 3) showed that, plant height in both seasons was significantly affected by sowing date. The shortest plants were produced by early planting, whereas the tallest plants were recorded by late sowing and the plant height tended to increase by delay planting date. The results indicated that late sown plants grew faster than early sown ones, which is evident that high temperature provided by late sowing enhanced stem elongation of cotton plants. Similar results were reported by El-Sayed (2005), Abd El-Twab (2006), Hakoomat et al. (2009) and Hayatullah et al. (2011). On the contrary, Ali and El-Sayed (2001), Munk (2001) and Wrather et al. (2008) reported that plant height was not significantly affected by planting date. Also, the present data showed that plant heights over the two seasons were significantly affected by growth regulator PIX at $150 \mathrm{ppm}$ gave the shortest plants, whereas the tallest plants were recorded by the promising at $30 \mathrm{ppm}$ NAA. The interaction between planting dates and growth regulators had a significant effect on plant height in both seasons where, the planting date in 25 May (late) and $30 \mathrm{ppm}$ NAA gave the highest values of plant height in both seasons.

\subsubsection{Days to first bud, flower and open boll}

The data obtained in Tables (1,2 and 3) revealed that, days from planting to the first bud, flower and open boll, were significantly affected by sowing dates, growth regulators and their interaction. Late planting brought the appearance of the first bud, first flower and first open boll early in the two growing seasons as compared to middle and early planting. This might be due to relatively low night temperature of early planting date at the beginning of the season which prolonged the period of the appearance of the first bud, flowering and bolling. Similar results were obtained by Emara et al. (2006), Abd El-Twab (2009) and Wenqing et al. (2012). Data also showed that the mean performance of PIX growth regulators at $50 \mathrm{ppm}$ was superior in phenologyical stage which gave the best reading in all No. of days to $1^{\text {st }}$ bud, $1^{\text {st }}$ flower and $1^{\text {st }}$ open boll $(23.11,26.18),(43.37,45.59)$ and $(90.04$, 95.44) in 2010 and 2011 seasons, respectively, Similar results were obtained by El-Sayed (2006) Zakaria et al. (2006) and Stephen et al. (2007). The differences between the use of $50 \mathrm{ppm}$ PIX or $50 \mathrm{ppm}$ IAA the not significant in the 1 st. open boll in the second season only. Use of $50 \mathrm{ppm}$ PIX with sowing at 25 March decreased No. of days to appearance of the $1^{s t}$ bud, the $1^{\text {st }}$ flower and the $1^{\text {st }}$ open boll in both seasons.

\subsubsection{Yield and yield component}

\subsubsection{Number of sympodial branches/plant}

Data in Tables $(4,5$ and 6$)$ showed that, the number of sympodial branches/plant was significantly affected by sowing date, growth regulators and the interaction between them. Early planting dates (25 March) increased the number of sympodial branches/plant as compared to middle and late planting. Similar results were obtained by William et al. (2012), Hakoomat et al. (2009) and Abd El-Twab (2009). Data also show that the growth regulator PIX at $50 \mathrm{ppm}$ exhibited high value of the number of sympodial branches/plant followed by IAA at $50 \mathrm{ppm}$ in the first season, while in the second season the use of 50 ppm IAA or $50 \mathrm{ppm}$ PIX did not differ significantly in the value of the No. of sympodial branches/plant. While the growth regulator NAA at $30 \mathrm{ppm}$ exhibited low value of mean performance of number of sympodial branches/plant in both seasons. The obtained results are in agreement with those recorded by Shahzad et al. (2012), Emara et al. (2009), Elayan. (2008 b), Sawan and 
Table (1): Main effects of sowing dates and growth regulator treatments on plant height and some phenologycal stages of cotton during 2010 \& 2011 seasons.

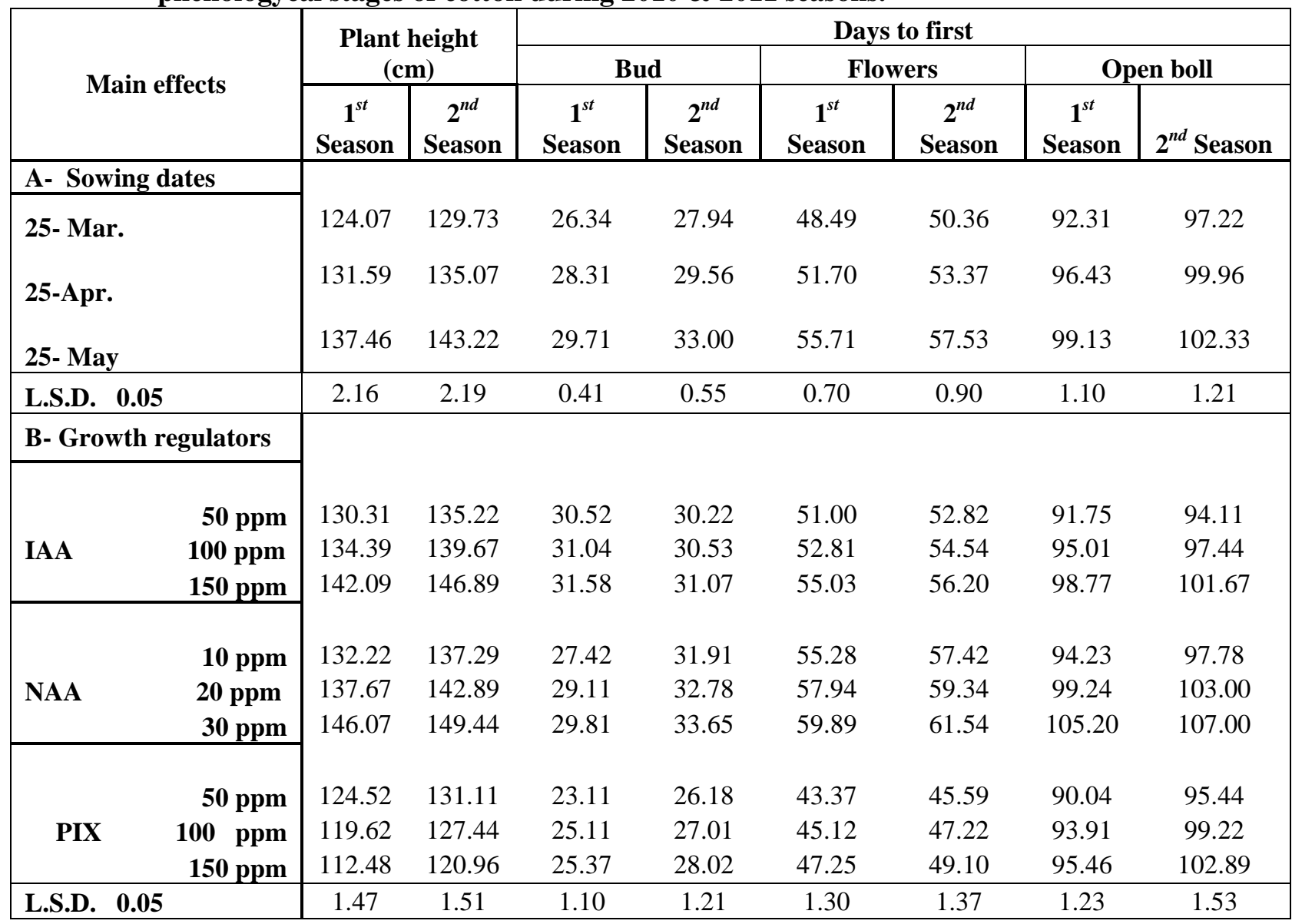

Sakr. (2008). The planting date in 25 March (early) and growth regulator (PIX) at $50 \mathrm{ppm}$ gave (early) and growth regulator (PIX) at $50 \mathrm{ppm}$ gave the highest values in the first season only, while in the second season planting in 25 March and the use of $50 \mathrm{ppm}$ IAA gave the highest value on the No. of sympodial branches/plant. These results are rather expected as the seed cotton yield / plant was increased by early planting.

\subsubsection{Number of open bolls per plant}

Data presented in Tables (4, 5 and 6) revealed that sowing date and growth regulators exerted significant effects on the number of open bolls/ plant in both seasons. There was a tendency of increasing the number of bolls per plant in favour of early planting as compared to middle and late plantings. Similar results were obtained by Zhiguo et al. (2011), Abd El-Twab (2009) and Elayan et al. (2006). The results indicted that the use of 50 ppm PIX exhibited the highest values of the number of bolls per plant $(16.84,17.41)(2010$ and 2011 seasons), while NAA 30 ppm exhibited the lowest value of mean performance of the number of open bolls per plant. The obtained results are in agreement with those of Sawan and Sakr (2008), Jonathan and Stewart (2006) and Stephen et al.
(2007). Early sowing (25 March) with $50 \mathrm{ppm}$ PIX or 100 ppm PIX did not significantly differ in the No. of open bolls/ plant in the second season only, while in the first season the use of $50 \mathrm{ppm}$ PIX with 25 March was superior in this trait.

\subsubsection{Boll weight}

Data presented in Tables $(4,5$ and 6$)$ showed that, boll weight was not significantly affected by sowing date, growth regulators in the two growing seasons. Delayed planting date from 25 March to 25 May decreased the boll weight. This could be due to that early sown plants were shorter than late sown ones and hence could partition more available photosynthetic, for boll growth and hence weight. Early sown plants also, afforded longer vegetative duration as was expressed in the larger number of days to flowering, therefore, more active photosynthates could have been preformed with relatively lower night temperature as well lower heat unit by early sown plants than by the late sown ones. Shorter plants are always having lower maintenance respiration than longer ones. The obtained results are in agreement with those recorded by Reddy et al. (1999), El-Sayed (2005), Emara et al. (2009) and Wenqing et al. (2012) and in disagreement with Boquet et al. 
Table (2): The interactions between sowing dates and growth regulator treatments on plant height and some phenologycal stages of cotton during 2010 season.

\begin{tabular}{|c|c|c|c|c|c|c|}
\hline \multicolumn{3}{|c|}{ Treatments } & \multirow[b]{2}{*}{$\begin{array}{l}\text { Plant height } \\
(\mathbf{c m})\end{array}$} & \multicolumn{3}{|c|}{ No. of Days to } \\
\hline $\begin{array}{c}\text { Sowing } \\
\text { dates(A) }\end{array}$ & $\begin{array}{r}\mathbf{G} \\
\text { regul }\end{array}$ & $\begin{array}{l}\text { owth } \\
\text { ators (B) }\end{array}$ & & $1^{s t}$ Bud & $1^{s t}$ Flower & $\begin{array}{c}1^{s t} \text { Open } \\
\text { boll }\end{array}$ \\
\hline \multirow{3}{*}{ 25-Mar. } & IAA & $\begin{array}{c}50 \mathrm{ppm} \\
100 \mathrm{ppm} \\
150 \mathrm{ppm}\end{array}$ & $\begin{array}{l}122.61 \\
129.45 \\
137.75\end{array}$ & $\begin{array}{l}29.34 \\
28.23 \\
28.00\end{array}$ & $\begin{array}{l}47.20 \\
49.01 \\
51.56\end{array}$ & $\begin{array}{l}88.13 \\
92.26 \\
95.30\end{array}$ \\
\hline & NAA & $\begin{array}{c}10 \text { ppm } \\
20 \text { ppm } \\
30 \text { ppm }\end{array}$ & $\begin{array}{l}125.12 \\
132.15 \\
141.21\end{array}$ & $\begin{array}{l}27.15 \\
26.52 \\
27.13\end{array}$ & $\begin{array}{l}53.12 \\
55.10 \\
57.24\end{array}$ & $\begin{array}{c}92.46 \\
97.10 \\
102.25\end{array}$ \\
\hline & PIX & $\begin{array}{r}50 \mathrm{ppm} \\
100 \mathrm{ppm} \\
150 \mathrm{ppm} \\
\end{array}$ & $\begin{array}{l}116.03 \\
110.10 \\
102.22\end{array}$ & $\begin{array}{l}22.52 \\
24.88 \\
23.30\end{array}$ & $\begin{array}{l}40.00 \\
41.20 \\
42.00\end{array}$ & $\begin{array}{l}84.71 \\
88.41 \\
90.16\end{array}$ \\
\hline \multirow{3}{*}{ 25-Apr. } & IAA & $\begin{array}{r}50 \mathrm{ppm} \\
100 \mathrm{ppm} \\
150 \mathrm{ppm}\end{array}$ & $\begin{array}{l}132.07 \\
133.26 \\
142.00\end{array}$ & $\begin{array}{l}30.11 \\
31.36 \\
32.01\end{array}$ & $\begin{array}{l}51.45 \\
52.23 \\
53.81\end{array}$ & $\begin{array}{l}92.00 \\
95.33 \\
99.00\end{array}$ \\
\hline & NAA & $\begin{array}{c}10 \mathrm{ppm} \\
20 \mathrm{ppm} \\
30 \mathrm{ppm}\end{array}$ & $\begin{array}{l}133.13 \\
136.44 \\
145.00\end{array}$ & $\begin{array}{l}26.56 \\
29.10 \\
30.11\end{array}$ & $\begin{array}{l}54.50 \\
57.30 \\
59.25\end{array}$ & $\begin{array}{c}94.11 \\
98.48 \\
105.00\end{array}$ \\
\hline & PIX & $\begin{array}{r}50 \mathrm{ppm} \\
100 \mathrm{ppm} \\
150 \mathrm{ppm} \\
\end{array}$ & $\begin{array}{l}125.00 \\
122.33 \\
115.11\end{array}$ & $\begin{array}{l}23.71 \\
25.25 \\
26.55\end{array}$ & $\begin{array}{l}42.10 \\
45.00 \\
49.66\end{array}$ & $\begin{array}{l}91.71 \\
95.00 \\
97.23\end{array}$ \\
\hline \multirow{3}{*}{ 25-May } & IAA & $\begin{array}{r}50 \mathrm{ppm} \\
100 \mathrm{ppm} \\
150 \mathrm{ppm} \\
\end{array}$ & $\begin{array}{l}136.25 \\
140.46 \\
146.51\end{array}$ & $\begin{array}{l}32.10 \\
33.52 \\
34.74\end{array}$ & $\begin{array}{l}54.36 \\
57.20 \\
59.73\end{array}$ & $\begin{array}{c}95.11 \\
97.45 \\
102.00\end{array}$ \\
\hline & NAA & $\begin{array}{c}10 \text { ppm } \\
20 \text { ppm } \\
30 \text { ppm }\end{array}$ & $\begin{array}{l}138.41 \\
144.43 \\
152.00\end{array}$ & $\begin{array}{l}28.54 \\
31.70 \\
32.20\end{array}$ & $\begin{array}{l}58.22 \\
61.43 \\
63.17\end{array}$ & $\begin{array}{c}96.11 \\
102.15 \\
108.36\end{array}$ \\
\hline & PIX & $\begin{array}{r}50 \mathrm{ppm} \\
100 \mathrm{ppm} \\
150 \mathrm{ppm}\end{array}$ & $\begin{array}{l}132.53 \\
126.43 \\
120.11\end{array}$ & $\begin{array}{l}23.10 \\
25.21 \\
26.25\end{array}$ & $\begin{array}{l}48.01 \\
49.17 \\
50.10\end{array}$ & $\begin{array}{l}93.70 \\
98.33 \\
99.00\end{array}$ \\
\hline \multicolumn{3}{|c|}{ L.S.D. 0.05} & 2.75 & 1.02 & 1.25 & 2.33 \\
\hline
\end{tabular}


Table (3): The interactions between sowing dates and growth regulator treatments on plant height and some phenologycal stages of cotton during 2011 season.

\begin{tabular}{|c|c|c|c|c|c|c|}
\hline \multicolumn{3}{|c|}{ Treatments } & \multirow{2}{*}{$\begin{array}{l}\text { Plant height } \\
\text { (cm) }\end{array}$} & \multicolumn{3}{|c|}{ No. of Days to } \\
\hline $\begin{array}{c}\text { Sowing } \\
\text { dates }(\mathrm{A})\end{array}$ & $\begin{array}{r}\text { C } \\
\text { regu }\end{array}$ & $\begin{array}{l}\text { owth } \\
\text { tors (B) }\end{array}$ & & $1^{s t}$ Bud & $1^{s t}$ Flower & $1^{s t}$ Open boll \\
\hline \multirow{3}{*}{ 25-Mar. } & IAA & $\begin{array}{r}50 \mathrm{ppm} \\
100 \mathrm{ppm} \\
150 \mathrm{ppm}\end{array}$ & $\begin{array}{l}129.67 \\
136.33 \\
143.33\end{array}$ & $\begin{array}{l}27.75 \\
28.10 \\
29.16\end{array}$ & $\begin{array}{l}49.66 \\
50.22 \\
52.20\end{array}$ & $\begin{array}{l}92.00 \\
95.00 \\
98.00\end{array}$ \\
\hline & NAA & $\begin{array}{c}10 \text { ppm } \\
20 \text { ppm } \\
30 \text { ppm }\end{array}$ & $\begin{array}{l}133.20 \\
138.00 \\
145.67\end{array}$ & $\begin{array}{l}30.10 \\
30.85 \\
31.25\end{array}$ & $\begin{array}{l}55.25 \\
57.14 \\
59.31\end{array}$ & $\begin{array}{c}95.00 \\
99.67 \\
104.67\end{array}$ \\
\hline & PIX & $\begin{array}{r}50 \mathrm{ppm} \\
100 \mathrm{ppm} \\
150 \mathrm{ppm} \\
\end{array}$ & $\begin{array}{l}120.33 \\
113.67 \\
107.33\end{array}$ & $\begin{array}{l}24.15 \\
24.90 \\
25.20\end{array}$ & $\begin{array}{l}42.14 \\
43.10 \\
44.20\end{array}$ & $\begin{array}{c}94.00 \\
96.33 \\
100.33\end{array}$ \\
\hline \multirow{3}{*}{ 25-Apr. } & IAA & $\begin{array}{r}50 \text { ppm } \\
100 \text { ppm } \\
150 \text { ppm }\end{array}$ & $\begin{array}{l}134.67 \\
136.00 \\
146.00\end{array}$ & $\begin{array}{l}30.78 \\
30.00 \\
29.80\end{array}$ & $\begin{array}{l}52.00 \\
54.10 \\
55.17\end{array}$ & $\begin{array}{c}93.67 \\
97.33 \\
102.67\end{array}$ \\
\hline & NAA & $\begin{array}{c}10 \mathrm{ppm} \\
20 \mathrm{ppm} \\
30 \mathrm{ppm}\end{array}$ & $\begin{array}{l}136.00 \\
141.00 \\
146.67\end{array}$ & $\begin{array}{l}31.41 \\
32.15 \\
33.40\end{array}$ & $\begin{array}{l}57.00 \\
58.33 \\
60.27\end{array}$ & $\begin{array}{c}97.67 \\
103.33 \\
107.00\end{array}$ \\
\hline & PIX & $\begin{array}{r}50 \mathrm{ppm} \\
100 \mathrm{ppm} \\
150 \mathrm{ppm} \\
\end{array}$ & $\begin{array}{l}130.00 \\
127.00 \\
118.33\end{array}$ & $\begin{array}{l}25.15 \\
26.20 \\
27.11\end{array}$ & $\begin{array}{l}45.13 \\
48.20 \\
50.10\end{array}$ & $\begin{array}{c}95.67 \\
99.33 \\
103.00\end{array}$ \\
\hline \multirow{3}{*}{ 25-May } & IAA & $\begin{array}{r}50 \mathrm{ppm} \\
100 \mathrm{ppm} \\
150 \mathrm{ppm} \\
\end{array}$ & $\begin{array}{l}141.33 \\
146.67 \\
151.33\end{array}$ & $\begin{array}{l}32.12 \\
33.50 \\
34.25\end{array}$ & $\begin{array}{l}56.80 \\
59.31 \\
61.22\end{array}$ & $\begin{array}{c}96.67 \\
100.00 \\
104.33\end{array}$ \\
\hline & NAA & $\begin{array}{c}10 \text { ppm } \\
20 \text { ppm } \\
30 \text { ppm }\end{array}$ & $\begin{array}{l}141.67 \\
149.67 \\
156.00\end{array}$ & $\begin{array}{l}34.23 \\
35.36 \\
36.30\end{array}$ & $\begin{array}{l}60.00 \\
62.55 \\
65.03\end{array}$ & $\begin{array}{l}100.67 \\
106.00 \\
109.33\end{array}$ \\
\hline & $\begin{array}{l}\text { PIX } \\
\text {.D. } 0\end{array}$ & $\begin{array}{l}50 \mathrm{ppm} \\
100 \mathrm{ppm} \\
150 \mathrm{ppm} \\
5\end{array}$ & $\begin{array}{c}143.00 \\
131.33 \\
127.00 \\
2.93\end{array}$ & $\begin{array}{c}29.23 \\
30.22 \\
31.75 \\
1.22\end{array}$ & $\begin{array}{r}49.50 \\
50.35 \\
53.00 \\
1.37\end{array}$ & $\begin{array}{c}96.67 \\
102.00 \\
105.33 \\
2.50\end{array}$ \\
\hline
\end{tabular}




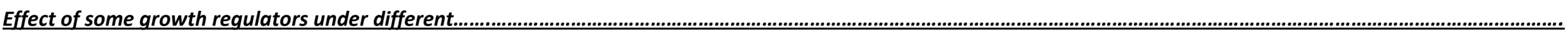

Table (4): Main effects of sowing dates and growth regulator treatments on yield and yield component characters in 2010 \&2011 seasons.

\begin{tabular}{|c|c|c|c|c|c|c|c|c|c|c|c|c|}
\hline \multirow{2}{*}{ Main effects } & \multicolumn{2}{|c|}{$\begin{array}{l}\text { Number of sympodial } \\
\text { branches /plant }\end{array}$} & \multicolumn{2}{|c|}{$\begin{array}{l}\text { Number of open } \\
\text { bolls /plant }\end{array}$} & \multicolumn{2}{|c|}{ Boll weight (gm.) } & \multicolumn{2}{|c|}{ Seed index (gm.) } & \multicolumn{2}{|c|}{ Earliness (\%) } & \multicolumn{2}{|c|}{$\begin{array}{l}\text { Seed cotton yield } \\
\quad(\text { Ken./fed })\end{array}$} \\
\hline & $\begin{array}{c}1^{s t} \\
\text { Season } \\
\end{array}$ & $\begin{array}{c}2^{n d} \\
\text { Season } \\
\end{array}$ & $\begin{array}{c}1^{\text {st }} \\
\text { Season } \\
\end{array}$ & $\begin{array}{c}2^{n d} \\
\text { Season }\end{array}$ & $\begin{array}{c}1^{s t} \\
\text { Season }\end{array}$ & $\begin{array}{c}2^{n d} \\
\text { Season }\end{array}$ & $\begin{array}{c}1^{s t} \\
\text { Season }\end{array}$ & $\begin{array}{c}2^{n d} \\
\text { Season } \\
\end{array}$ & $\begin{array}{c}1^{s t} \\
\text { Season }\end{array}$ & $\begin{array}{c}2^{n d} \\
\text { Season }\end{array}$ & $\begin{array}{c}1^{s t} \\
\text { Season }\end{array}$ & $\begin{array}{c}2^{n d} \\
\text { Season }\end{array}$ \\
\hline \multicolumn{13}{|l|}{$\begin{array}{c}\text { A- Sowing } \\
\text { dates }\end{array}$} \\
\hline \multirow{3}{*}{$\begin{array}{l}\text { 25-Mar. } \\
\text { 25-Apr. }\end{array}$} & 15.36 & 16.54 & 15.26 & 16.17 & 2.37 & 2.48 & 11.86 & 12.44 & 62.44 & 64.14 & 11.68 & 12.37 \\
\hline & 13.62 & 15.93 & 13.07 & 14.08 & 2.30 & 2.42 & 11.04 & 11.43 & 61.37 & 62.78 & 11.12 & 11.54 \\
\hline & 11.59 & 12.74 & 11.52 & 12.45 & 2.26 & 2.37 & 10.37 & 10.95 & 59.95 & 61.39 & 10.54 & 11.00 \\
\hline \begin{tabular}{ll|} 
L.S.D. & 0.05 \\
\end{tabular} & 0.46 & 0.52 & 1.61 & 1.73 & N.S. & N.S. & 1.43 & 1.55 & 0.62 & 0.74 & 0.55 & 0.68 \\
\hline \multicolumn{13}{|l|}{$\begin{array}{l}\text { B- Growth } \\
\text { regulators }\end{array}$} \\
\hline \multirow{3}{*}{$\begin{array}{cc} & 50 \mathrm{ppm} \\
\text { IAA } & 100 \mathrm{~m} \\
150 \mathrm{ppm} \\
\end{array}$} & 15.32 & 17.18 & 15.86 & 16.95 & 2.44 & 2.55 & 11.33 & 11.97 & 62.64 & 64.27 & 11.83 & 12.62 \\
\hline & 13.87 & 15.53 & 13.35 & 14.33 & 2.24 & 2.38 & 10.81 & 11.27 & 61.4 & 62.98 & 10.99 & 11.5 \\
\hline & 11.89 & 13.71 & 10.87 & 12.19 & 2.15 & 2.25 & 10.16 & 10.53 & 59.48 & 61.29 & 10.60 & 10.73 \\
\hline \multirow{3}{*}{$\begin{array}{cc} & 10 \mathrm{ppm} \\
\text { NAA } & 20 \mathrm{pm} \\
& 30 \mathrm{ppm}\end{array}$} & 14.59 & 16.04 & 13.94 & 15.35 & 2.32 & 2.47 & 10.76 & 11.26 & 61.32 & 63.61 & 11.04 & 11.53 \\
\hline & 11.86 & 14.29 & 12.39 & 13.32 & 2.14 & 2.36 & 10.02 & 10.72 & 59.83 & 61.5 & 10.31 & 10.83 \\
\hline & 10.52 & 12.72 & 10.25 & 11.22 & 2.03 & 2.11 & 9.64 & 10.18 & 58.50 & 60.44 & 9.60 & 10.32 \\
\hline \multirow{3}{*}{$\begin{array}{l}\text { PIX } 100 \text { ppm } \\
150 \text { ppm }\end{array}$} & 16.37 & 17.14 & 16.84 & 17.41 & 2.58 & 2.65 & 12.72 & 13.55 & 60.04 & 65.08 & 12.72 & 13.32 \\
\hline & 14.60 & 15.38 & 13.94 & 14.66 & 2.48 & 2.61 & 12.06 & 12.74 & 62.84 & 63.96 & 11.86 & 12.34 \\
\hline & 12.67 & 13.65 & 12.09 & 12.67 & 2.39 & 2.48 & 11.75 & 12.24 & 61.21 & 61.83 & 11.07 & 11.51 \\
\hline L.S.D. 0.05 & 0.39 & 0.47 & 1.55 & 1.67 & N.S. & N.S. & 1.85 & 1.96 & 0.33 & 0.47 & 0.44 & 0.61 \\
\hline
\end{tabular}


Table (5): The interactions between sowing dates and growth regulator treatments on yield and yield component characters 2010 season.

\begin{tabular}{|c|c|c|c|c|c|c|c|c|}
\hline \multicolumn{3}{|c|}{ Treatments } & \multirow{2}{*}{$\begin{array}{c}\text { Number } \\
\text { of } \\
\text { sympodial } \\
\text { branches } \\
\text { /plant } \\
\end{array}$} & \multirow{2}{*}{$\begin{array}{c}\text { Number } \\
\text { of open } \\
\text { bolls } \\
\text { /plant }\end{array}$} & \multirow[b]{2}{*}{$\begin{array}{c}\text { Boll } \\
\text { weight } \\
\text { (gm.) }\end{array}$} & \multirow[b]{2}{*}{$\begin{array}{c}\text { Seed } \\
\text { index } \\
(\text { gm. })\end{array}$} & \multirow[b]{2}{*}{$\begin{array}{c}\text { Earliness } \\
(\%)\end{array}$} & \multirow[b]{2}{*}{$\begin{array}{c}\text { Seed cotton } \\
\text { yield } \\
\text { (Ken./fed) }\end{array}$} \\
\hline $\begin{array}{l}\text { Sowing } \\
\text { dates } \\
\text { (A) }\end{array}$ & $\begin{array}{r}\text { G } \\
\text { regu }\end{array}$ & $\begin{array}{l}\text { rowth } \\
\text { lators (B) }\end{array}$ & & & & & & \\
\hline \multirow{9}{*}{ 25-Mar. } & & 50 ppm & 17.10 & 18.43 & 2.52 & 11.83 & 63.80 & 12.55 \\
\hline & IAA & 100 ppm & 16.30 & 15.11 & 2.29 & 11.35 & 62.17 & 11.27 \\
\hline & & $150 \mathrm{ppm}$ & 13.81 & 12.23 & 2.18 & 10.52 & 60.63 & 10.92 \\
\hline & & $10 \mathrm{ppm}$ & 16.50 & 16.13 & 2.38 & 11.50 & 62.25 & 11.24 \\
\hline & NAA & $20 \mathrm{ppm}$ & 13.05 & 14.61 & 2.17 & 10.20 & 61.17 & 10.89 \\
\hline & & $30 \mathrm{ppm}$ & 11.70 & 11.72 & 2.07 & 10.00 & 59.67 & 10.09 \\
\hline & & 50 ppm & 18.45 & 19.50 & 2.68 & 13.76 & 65.50 & 13.45 \\
\hline & PIX & 100 ppm & 17.10 & 16.21 & 2.56 & 13.10 & 64.10 & 12.87 \\
\hline & & $150 \mathrm{ppm}$ & 14.23 & 13.44 & 2.44 & 12.86 & 62.63 & 11.89 \\
\hline \multirow{9}{*}{ 25-Apr. } & & 50 ppm & 15.75 & 15.46 & 2.41 & 11.17 & 62.67 & 11.89 \\
\hline & IAA & 100 ppm & 13.88 & 13.30 & 2.23 & 10.89 & 61.93 & 11.15 \\
\hline & & $150 \mathrm{ppm}$ & 11.77 & 10.86 & 2.14 & 10.01 & 59.36 & 10.77 \\
\hline & & $10 \mathrm{ppm}$ & 14.56 & 13.53 & 2.31 & 10.95 & 61.40 & 11.00 \\
\hline & NAA & $20 \mathrm{ppm}$ & 12.31 & 12.23 & 2.14 & 10.30 & 60.07 & 10.10 \\
\hline & & $30 \mathrm{ppm}$ & 10.85 & 10.41 & 2.02 & 9.93 & 58.50 & 9.80 \\
\hline & & $50 \mathrm{ppm}$ & 16.33 & 16.51 & 2.56 & 12.55 & 64.23 & 12.85 \\
\hline & PIX & 100 ppm & 14.45 & 13.46 & 2.46 & 12.01 & 62.97 & 11.70 \\
\hline & & $150 \mathrm{ppm}$ & 12.67 & 11.83 & 2.39 & 11.51 & 61.20 & 10.79 \\
\hline \multirow{9}{*}{ 25-May } & & 50 ppm & 13.11 & 13.70 & 2.38 & 11.00 & 61.47 & 11.04 \\
\hline & IAA & 100 ppm & 11.43 & 11.65 & 2.20 & 10.20 & 60.10 & 10.56 \\
\hline & & $150 \mathrm{ppm}$ & 10.10 & 9.51 & 2.12 & 9.96 & 58.43 & 10.12 \\
\hline & & $10 \mathrm{ppm}$ & 12.72 & 12.15 & 2.26 & 9.84 & 60.32 & 10.89 \\
\hline & NAA & $20 \mathrm{ppm}$ & 10.22 & 10.33 & 2.12 & 9.55 & 58.27 & 9.93 \\
\hline & & $30 \mathrm{ppm}$ & 9.00 & 8.63 & 2.00 & 9.00 & 57.33 & 8.91 \\
\hline & & $50 \mathrm{ppm}$ & 14.34 & 14.52 & 2.50 & 11.85 & 62.40 & 11.87 \\
\hline & PIX & 100 ppm & 12.26 & 12.15 & 2.42 & 11.08 & 61.47 & 11.00 \\
\hline & & 150 ppm & 11.12 & 11.00 & 2.35 & 10.89 & 59.80 & 10.53 \\
\hline \multicolumn{3}{|c|}{ L.S.D. 0.05} & 0.78 & 1.35 & 0.22 & 1.35 & 3.70 & 1.80 \\
\hline
\end{tabular}


Table (6): The interactions between sowing dates and growth regulator treatments on yield and yield component characters 2011 season.

\begin{tabular}{|c|c|c|c|c|c|c|c|c|}
\hline \multicolumn{3}{|c|}{ Treatments } & \multirow{2}{*}{$\begin{array}{l}\text { Number } \\
\text { of } \\
\text { sympodial } \\
\text { branches } \\
\text { /plant }\end{array}$} & \multirow{2}{*}{$\begin{array}{c}\text { Number } \\
\text { of open } \\
\text { bolls } \\
\text { /plant }\end{array}$} & \multirow[b]{2}{*}{$\begin{array}{c}\text { Boll } \\
\text { weight } \\
\text { (gm.) }\end{array}$} & \multirow[b]{2}{*}{$\begin{array}{c}\text { Seed } \\
\text { index } \\
\text { (gm.) }\end{array}$} & \multirow[b]{2}{*}{$\begin{array}{c}\text { Earliness } \\
(\%)\end{array}$} & \multirow[b]{2}{*}{$\begin{array}{c}\text { Seed cotton } \\
\text { yield } \\
\text { (Ken./fed) }\end{array}$} \\
\hline $\begin{array}{c}\text { Sowing } \\
\text { dates } \\
\text { (A) }\end{array}$ & $\begin{array}{r}\mathbf{G} \\
\text { regul }\end{array}$ & $\begin{array}{l}\text { rowth } \\
\text { ators (B) }\end{array}$ & & & & & & \\
\hline \multirow{9}{*}{ 25-Mar. } & & 50 ppm & 19.43 & 19.80 & 2.62 & 12.85 & 65.50 & 13.22 \\
\hline & IAA & 100 ppm & 18.25 & 17.10 & 2.42 & 11.75 & 64.07 & 12.35 \\
\hline & & $150 \mathrm{ppm}$ & 15.70 & 14.71 & 2.29 & 11.10 & 62.03 & 11.11 \\
\hline & & $10 \mathrm{ppm}$ & 18.41 & 17.13 & 2.56 & 11.92 & 64.83 & 12.33 \\
\hline & NAA & $20 \mathrm{ppm}$ & 16.36 & 15.56 & 2.41 & 11.00 & 63.80 & 11.56 \\
\hline & & 30 ppm & 14.27 & 12.36 & 2.14 & 10.52 & 61.70 & 10.92 \\
\hline & & 50 ppm & 17.73 & 19.15 & 2.72 & 14.85 & 66.40 & 14.10 \\
\hline & PIX & 100 ppm & 15.52 & 16.30 & 2.67 & 14.10 & 65.20 & 13.10 \\
\hline & & $150 \mathrm{ppm}$ & 13.20 & 13.45 & 2.52 & 13.85 & 63.77 & 12.60 \\
\hline \multirow{9}{*}{ 25-Apr. } & & 50 ppm & 17.80 & 16.33 & 2.54 & 11.86 & 64.30 & 12.84 \\
\hline & IAA & 100 ppm & 16.12 & 13.33 & 2.37 & 11.10 & 63.00 & 11.40 \\
\hline & & 150 ppm & 14.32 & 11.82 & 2.25 & 10.38 & 61.43 & 10.98 \\
\hline & & $10 \mathrm{ppm}$ & 16.25 & 15.45 & 2.46 & 11.00 & 63.53 & 11.31 \\
\hline & NAA & 20 ppm & 14.21 & 13.16 & 2.36 & 10.86 & 61.43 & 10.93 \\
\hline & & 30 ppm & 13.45 & 11.81 & 2.11 & 10.02 & 60.90 & 10.20 \\
\hline & & 50 ppm & 18.55 & 17.86 & 2.64 & 13.50 & 65.47 & 13.01 \\
\hline & PIX & 100 ppm & 17.16 & 14.52 & 2.60 & 12.25 & 63.90 & 12.10 \\
\hline & & 150 ppm & 15.48 & 12.46 & 2.48 & 11.87 & 61.07 & 11.07 \\
\hline \multirow{9}{*}{ 25-May } & & 50 ppm & 14.31 & 14.73 & 2.48 & 11.20 & 63.00 & 11.80 \\
\hline & IAA & 100 ppm & 12.22 & 12.57 & 2.34 & 10.95 & 61.87 & 10.75 \\
\hline & & 150 ppm & 11.11 & 10.05 & 2.21 & 10.10 & 60.40 & 10.10 \\
\hline & & 10 ppm & 13.45 & 13.46 & 2.38 & 10.86 & 62.47 & 10.95 \\
\hline & NAA & $20 \mathrm{ppm}$ & 12.30 & 11.24 & 2.31 & 10.30 & 59.27 & 10.01 \\
\hline & & 30 ppm & 10.44 & 9.50 & 2.07 & 10.00 & 58.73 & 9.85 \\
\hline & & $50 \mathrm{ppm}$ & 15.13 & 15.21 & 2.60 & 12.30 & 63.37 & 12.86 \\
\hline & PIX & 100 ppm & 13.45 & 13.15 & 2.55 & 11.86 & 62.77 & 11.81 \\
\hline & & $150 \mathrm{ppm}$ & 12.27 & 12.10 & 2.43 & 11.00 & 60.67 & 10.86 \\
\hline \multicolumn{3}{|c|}{ L.S.D. 0.05} & 0.97 & 1.20 & 0.41 & 1.51 & 3.92 & 1.93 \\
\hline
\end{tabular}


Table (7): Main effects of sowing dates and growth regulator treatments on fiber properties of cotton during $2010 \& 2011$ seasons.

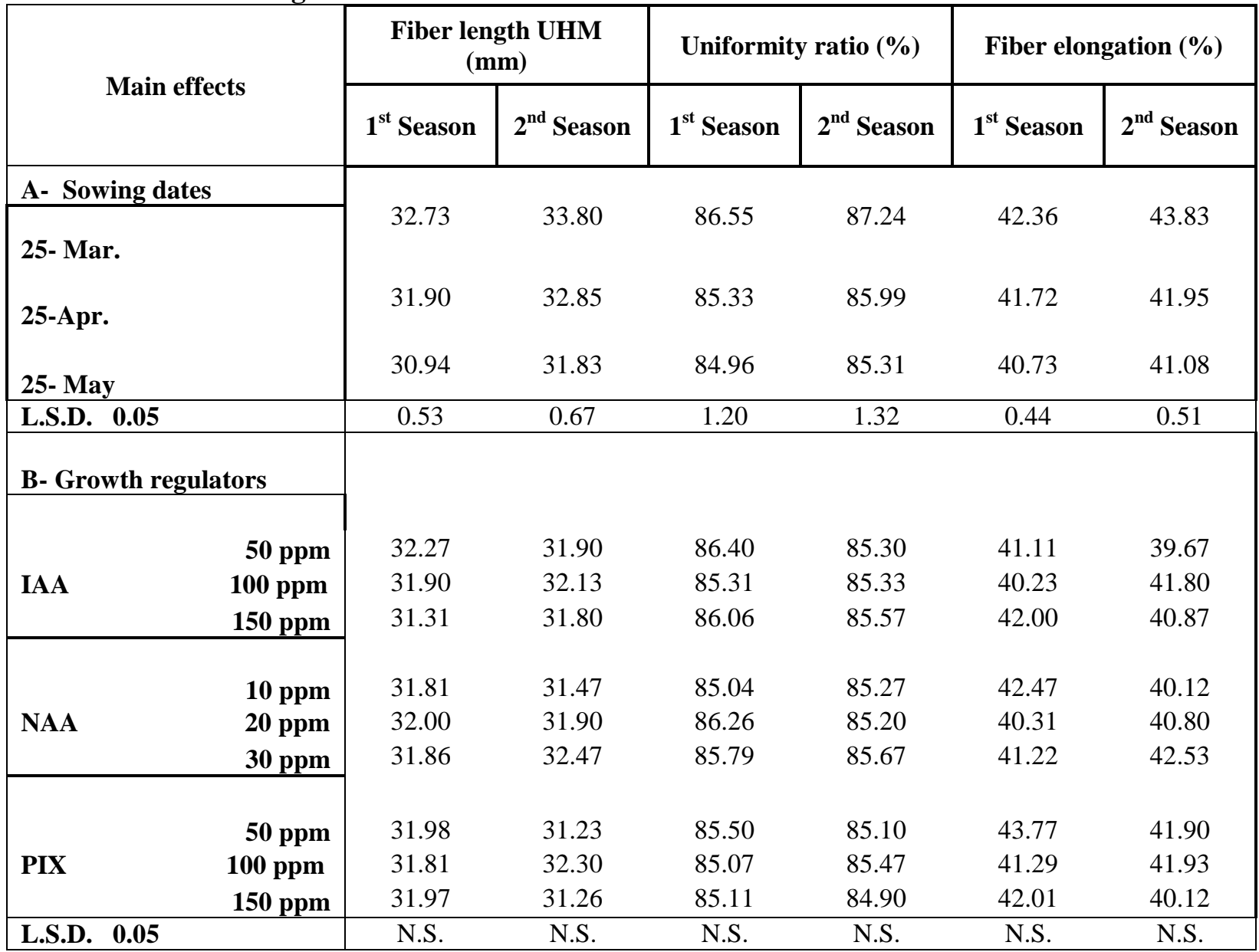

(2003). The present data also show that the growth regulator PIX at $50 \mathrm{ppm}$ followed by IAA $50 \mathrm{ppm}$ exhibited high value and did not differ significantly in the mean performance of boll weight $(2.58,2.65)$ and $(2.44,2.55)$ in $(2010$ and 2011), respectively, while NAA at $30 \mathrm{ppm}$ exhibited lower value of mean performance of boll weight. Similar results were obtained by Zakaria et al. (2006), Sawan and Sakr (2008) and Emara et al. (2009).The planting date in 25 March (early) and 50 ppm PIX gave the highest values of this character.

\subsubsection{Seed index}

Data in Tables (4, 5 and 6) showed that, the seed index was significantly affected by sowing date, growth regulators and the interaction between them in the two seasons. Delaying planting from 25 March to 25 May decreased seed index, where early planting gave the highest values of seed index as compared to middle and late plantings. Seeds in early planting had more available photosynthates to filling than late sown ones. Similar results were obtained by Abd ElTwab (2009), Hakoomat et al. (2009) and Zhiguo et al. (2011). Data also show that the growth regulator PIX at $50 \mathrm{ppm}$ followed by IAA at 50 ppm exhibited the highest values of mean performance of seed index, while NAA at $30 \mathrm{ppm}$ exhibited lower value of mean performance of seed index. The obtained results are in agreement with those of Emara et al. (2009) and Shahzad et al. (2012). Use of $50 \mathrm{ppm}$ PIX with 25 March or 25 April did not differ significantly in both seasons and gave the highest values in this character.

\subsubsection{Earliness percentage}

Data in Tables (4, 5 and 6) showed that, earliness \% was significantly affected by sowing date, growth regulators and their interaction;

earliness percentage was decreased by delaying the date of planting. This might be due to early flowering and hence early boll maturity which was expressed in fewer numbers of days to flowering and to first open boll compared to late planted cotton. Similar results were obtained by Hayatullah et al. (2011), Zhiguo et al. (2011), and Wenqing et al. (2012). Concerning growth regulators, the data showed that, use of $50 \mathrm{ppm}$ PIX was superior in earliness (\%) in the second season only, while the use of 100 ppm PIX was 
Table (8): Main effects of sowing dates and growth regulator treatments on fiber properties of cotton during 2010 \& 2011 seasons.

\begin{tabular}{|c|c|c|c|c|c|c|c|c|c|}
\hline \multirow{3}{*}{\multicolumn{2}{|c|}{ Main effects }} & \multirow{2}{*}{\multicolumn{2}{|c|}{$\begin{array}{l}\text { Fiber strength } \\
\quad(\text { gem/ tex })\end{array}$}} & \multirow{2}{*}{\multicolumn{2}{|c|}{ Microniare reading }} & \multicolumn{4}{|c|}{ Values of color } \\
\hline & & & & & & \multicolumn{2}{|c|}{ Brightness } & \multicolumn{2}{|c|}{ Yellowness } \\
\hline & & $\begin{array}{c}1^{\text {st }} \\
\text { Season }\end{array}$ & $\begin{array}{c}2^{\text {nd }} \\
\text { Season }\end{array}$ & $\begin{array}{c}1^{\text {st }} \\
\text { Season }\end{array}$ & $\begin{array}{c}2^{\text {nd }} \\
\text { Season }\end{array}$ & $\begin{array}{c}1^{\text {st }} \\
\text { Season }\end{array}$ & $\begin{array}{c}2^{\text {nd }} \\
\text { Season }\end{array}$ & $\begin{array}{c}1^{s t} \\
\text { Season }\end{array}$ & $\begin{array}{c}2^{\text {nd }} \\
\text { Season }\end{array}$ \\
\hline \multicolumn{2}{|c|}{ A- Sowing dates } & & & & & & & & \\
\hline \multicolumn{2}{|c|}{ 25-Mar. } & 7.38 & 7.48 & 3.00 & 2.78 & 71.46 & 73.71 & 9.04 & 8.41 \\
\hline \multicolumn{2}{|c|}{ 25-Apr. } & 6.94 & 7.21 & 3.27 & 3.07 & 71.20 & 73.60 & 9.45 & 8.92 \\
\hline \multicolumn{2}{|c|}{ 25-May } & 6.73 & 7.07 & 3.40 & 3.26 & 69.12 & 70.61 & 9.91 & 9.53 \\
\hline \multicolumn{2}{|c|}{$\begin{array}{l}\text { L.S.D. } 0.05 \\
\end{array}$} & 0.12 & 0.14 & 0.26 & 0.37 & 0.11 & 0.45 & 0.23 & 0.30 \\
\hline \multicolumn{2}{|c|}{ B- Growth regulators } & & & & & & & & \\
\hline \multirow{3}{*}{ IAA } & $50 \mathrm{ppm}$ & 7.22 & 7.40 & 3.32 & 3.15 & 70.74 & 72.55 & 9.54 & 8.82 \\
\hline & 100 ppm & 7.25 & 6.50 & 3.15 & 3.17 & 70.53 & 72.98 & 9.32 & 8.62 \\
\hline & $150 \mathrm{ppm}$ & 6.14 & 7.67 & 3.22 & 3.20 & 69.78 & 73.57 & 9.69 & 8.92 \\
\hline \multirow{3}{*}{ NAA } & 10 ppm & 7.01 & 6.10 & 3.21 & 3.20 & 70.63 & 72.36 & 9.37 & 8.99 \\
\hline & $20 \mathrm{ppm}$ & 7.36 & 7.63 & 3.33 & 3.07 & 71.00 & 73.52 & 9.65 & 9.18 \\
\hline & 30 ppm & 7.08 & 7.05 & 3.20 & 3.94 & 70.60 & 72.29 & 9.30 & 9.05 \\
\hline \multirow{3}{*}{ PIX } & $50 \mathrm{ppm}$ & 7.24 & 7.00 & 3.16 & 3.27 & 70.70 & 72.61 & 9.51 & 8.86 \\
\hline & $100 \mathrm{ppm}$ & 6.94 & 7.03 & 3.22 & 3.22 & 70.31 & 71.31 & 9.29 & 9.07 \\
\hline & $150 \mathrm{ppm}$ & 6.92 & 7.22 & 3.22 & 3.17 & 71.04 & 72.57 & 9.53 & 9.04 \\
\hline \multicolumn{2}{|c|}{\begin{tabular}{|l|l|} 
L.S.D. & 0.05 \\
\end{tabular}} & N.S. & N.S. & N.S. & N.S. & N.S. & N.S. & N.S. & N.S. \\
\hline
\end{tabular}

superior in the first season. Use of $50 \mathrm{ppm}$ PIX with sowing at 25 March did not differ significant with sowing at 25 April in this trait in both seasons.

Similar results were obtained by Zakaria et al. (2006), Elayan (2008 b) and Shahzad et al. (2012). The planting date in 25 March (early) and PIX at $50 \mathrm{ppm}$ growth regulator gave the highest values of this character.

\subsubsection{Seed cotton yield per feddan}

Data in Tables (4, 5 and 6) showed that, seed cotton yield per feddan was significantly affected by sowing date, growth regulators and the interaction between them. Early planting dates increased seed cotton yield per feddan. This increase is mainly due to the increase of the number of open bolls per plant and increase in boll weight. Increased yield was recorded at early planting date as compared to middle and late planting. Similar results were obtained by Abd ElTwab (2009), Wenqing et al. (2012) and William et al. (2012). Data also show that the growth regulator PIX at $50 \mathrm{ppm}$ gave the highest value of seed cotton yield /fed., in both seasons followed by IAA at $50 \mathrm{ppm}$, while the growth regulator
NAA at 30 ppm exhibited a lower value of mean performance of seed cotton yield /fed. The obtained results are in agreement with those recorded by Stephen and Hensley (2007), Emara et al. (2009) and Shahzad et al. (2012). The planting date in 25 March (early) and growth regulator PIX at $50 \mathrm{ppm}$ gave the highest values in this character in the first season, while in the second season the use of 50 ppm PIX with sowing at 25 March did not differ significantly with sowing at 25 April. These results are rather expected as the seed cotton yield / plant was increased by early planting.

\subsubsection{Fiber properties}

Fiber characters include length measurements fiber length (UHM) and uniformity index, fiber bundle tensile properties (fiber strength and elongation) micronaire reading and color attribute values ( $\mathrm{Rd} \%$ and $+\mathrm{b})$. The mean values of sowing date and growth regulators in the two seasons 2010 and 2011 are presented in Tables (7 and 8). Sowing date had a significant effect on fiber properties whereas planting cotton in early date (25 March) gave the highest values in fiber length, uniformity ratio, fiber elongation, fiber strength 
and the best in micronire reading in both seasons. Fiber properties were decreased by delaying planting date. Similar results were obtained by Emara et al. (2006), Elayan et al. (2008 a), Zhiguo et al. (2011) and Wenqing et al. (2012). Data also showed that growth regulators had no significant effect on fiber properties in the two seasons. Similar results were obtained by Wahdan (2000), Frank et al. (2005), El-Sayed (2006), David and James (2008), Sawan and Sakr (2008), Emara et al. (2009) and Shahzad et al. (2012) and on the contrary, Steve et al. (2003) and Johnson (2005) indicated that mepiquate chloride increased fiber length, fiber elongation, maturity ratio and micronaire reading.

\section{REFERENCES}

A.S.T.M. (1998). (Designation D.4605-98 and 3818-98) Vol. 7. Mo1 Easton, MD, U.S.A.

Abdel-Twab, Rania M. (2009). Study of technological properties of some new Egyptian cotton varieties cultivated under different environmental conditions. M.Sc. Thesis, Fac. Agric., Cairo Univ., Egypt.

Abdel-Twab S.F. (2006). Effect of Climatic Conditions on Bolling, Earliness, Yield and Fiber Technology in cotton. Ph.D. Thesis, Fac. Agric., Cairo Univ., Egypt.

Ali, S.A. and El-Sayed A.E. (2001). Effect of sowing dates and nitrogen levels on growth, earliness and yield of Egyptian cotton cultivar Giza 88. Egypt. J. Agric. Res. 79 (1):221-232.

Boquet, D.J., Caylor, J. and Shivers, C. (2003) No-till cotton response to planting date. Proc. of Beltwide Cotton Conf. Nashville, TN. U.S.A. (6-10 Jan.,) (2): 2045-2047.

David G.R. and James L.F. (2008). Study of some growth regulators and yield of narrowrow cotton as affected by growth regulators. Agron. J. 94: 1317-1323.

Elayan Sohair E.D. (2008). Effect of some growth regulators on growth, yield and fibber properties of Giza 85 cotton variety. Egypt. J. of Appl. Sci, 23 (6A): 105-115.

Elayan Sohair E. D., Abd El-Hafeez A.A., Awad H.Y. and Hamoda S.A.F. (2006). Effect of light intensity and heat units on earliness, yield and fiber characters of cotton varieties. Egypt, J.Agric.Sci.Mansora Univ., 31 (7): 4107- 4118.

El-Sayed E.A. (2005). Effect of sowing and thinning dates on growth and yield of cotton J.

Agric. Sci. Moshtohor Univ. 30 (1): 41 -48.

El-Sayed E.A. (2006). Effect of Indol Acetic Acid (IAA) and Mepiquate chloride (PIX) application under different planting date on growth and yield in cotton. Egypt.J. Agric. Res., 84 (2):102-113.

Emara M.A., Elbagory Olfat H., El-Marakby A.M. and Makram E.A. (2006). The effect of planting date in relation to heat unit requirements on growth, yield and some fiber properties of cotton. Res. Bull., Fac. of Agric., Ain Shams Univ., 19:1-10.

Emara M.A, Makram E.A. Elbagory Olfat H., and El-Marakby A.M. (2009). Effect of mepiquate chloride and nitrogen fertilization application time on the Egyptian cotton cultivar Giza 80. Egypt.J. Agric. Res., 87 (1):187 -202.

Frank G., Bill R. and Rock L. (2005). Evaluation of mepiquat chloride treatments at cutout or the latest possible cutout date. Proc. Beltwide Cotton Conf., New Orleans LA., U.S.A., (Jan. 4-7) (2): 2161.

Hakoomat A., Muhammad N. A., Shakeel A. and Dilbaugh, M. (2009). Effect of cultivars and sowing dates on yield and quality of (Gossypium hirsotum). J. of Food, Agri., (7): 244-247.

Hayatullah, A., Inayatullah, A., Muhammad, M., Ejaz, A. K. and Muhammad, A. K. (2011). Effect of sowing time and plant spacing on fiber quality and seed cotton. Sarhad J. Agric. (27): 411-413.

Johnson J. T. (2005). Effects of mepiquat pentaborate on genotypes of varying maturity. Proc. Beltwide Cotton Conf., New Orleans LA. U.S.A., (Jan. 4-7) (1): 885.

Jonathan D. S. and Stewart A. M. (2006). Influence of plant density on cotton response to Mepiquat Chloride application. Agron. J. 98 (6): 1634-1639.

Munk D.S. (2001). Plant density and planting date impacts on Pima cotton development. University of California Cooperative Extension, Fresno County, Fresno, California U.S.A. 15 (5):32-43.

Reddy K. R., Davidonis G. H., Johnson A. S. and Vinyared B. T. (1999). Temperature regime and carbonioxide enrichment alter cotton boll development and fiber properties. Agron. J., 91 (5): $851-858$.

Sawan Z. M. and Sakr R. A. (2008). Effect of 1Naphthalene acetic acid concentrations and the number of its applications on the yield components, yield and fiber properties of Egyptian cotton (Gossypium barbadense L.). Crop Sci., 181 (2): 89-94.

Shahzad G.H., Rustamani M.A. and Shafi M. N. (2012). Effect of application of plant growth 
regulators on Earias vittella (Fabricius), infestation and yield components of Cotton. App. Sci., (8): 677-682.

Sndecor G.W. and Cochran W.G. (1981). Statistical methods. College press, Ames, Iowa, U.S.A.

Stephen P. k. and Hensley E. K. (2007). A reliable fruit set enhancer for cotton. Proc. Beltwide Cotton Conf., New Orleans, LA. U.S.A., (Jan. 9-12): 1112-1117.

Steve P. N., Charles E. S. and Mike A. J. (2003). Evaluation of row spacing and mepiquat chloride in cotton. J. Cotton Sci., 7: 148-155.

Wahdan A.A. (2000). Influence of growth regulators on yield and fiber quality in cotton. Crop Sci., 144 (2): 80-92.

Wenqing Z., Wang Y., Shu H.J. and Zhou Z. (2012). Sowing date and boll position affected boll weight, fiber quality and fiber physiological parameters in two cotton
(Gossypium hirsutum L.) cultivars. African J. of Agric., Res. 7(45): 6073-6081.

William T., Pettigrew R. and Meredith Jr. (2012). Seed quality and planting date effects on cotton lint yield, yield components, and fiber quality. Agron. and Soils 140 (10): 37-47.

Wrather J., Phipps B., Stevens W. and Phillips A. (2008). Cropping systems and water quality research. J. of Cotton Sci., 12 (1):1-7.

Zakaria S. M., Hafez S. A., Basyony A.E. and Alkassas A. R. (2006). Cottonseed, protein, oil yields and oil properties as affected by nitrogen fertilization and foliar application of potassium and a plant growth retardant. World J. Agric. Sci., 2 (1): 56-65.

Zhiguo Z. Y., Meng W. Y., Oosterhuis D. M. and Shu H. (2011). Effect of planting date and boll position on fiber strength of cotton (Gossypium hirsutum L.).Amer.,J. of Experimental Agric., 1(4): 331-342.

$$
\begin{aligned}
& \text { تأثير بعض منظمات النمو تحت مواعيد زراعة مختلفة علي النمو و المحصول فى القطن } \\
& \text { سهير عليان دسوقي عليان - أماني عحم عبد الله - * محه عبد الرحمن السيد - * رانيا عحم عبد التواب } \\
& \text { قسم المحاصبل- كلية الزر اعة ـ جامعة القاهرة و * قسم الغزل- معهد بحوث القطن ـ مركز البحوث الزراعية ـ الجيزة } \\
& \text { ملخص } \\
& \text { أجري هذا البحث بمحطة التجارب و البحوث الزراعية بكلية الزر اعة جامعة القاهرة خلال موسمي ( 2010- } 2011 \text { (IAA) }
\end{aligned}
$$

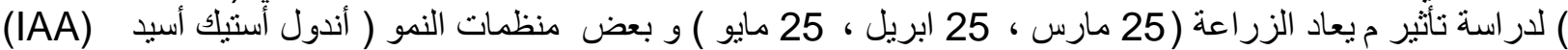

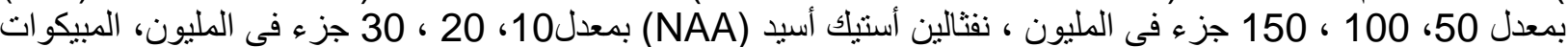
كلوريد (PIX)

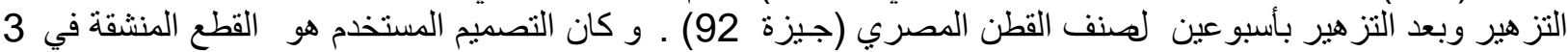

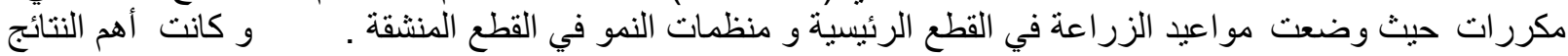

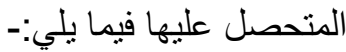
أثز ميعاد الزر اعة معنويا في كلا من الصفات الخضرية و المحصول و و مكوناته و الصفات التكنولوجية حيث ادي تأخير

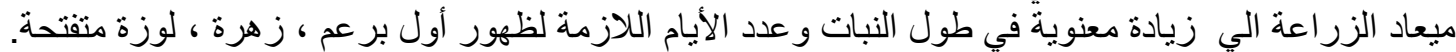

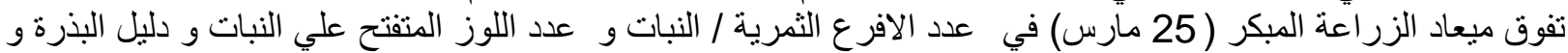

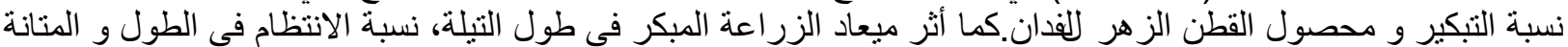

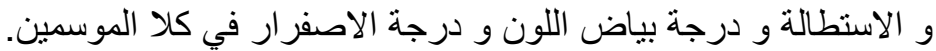

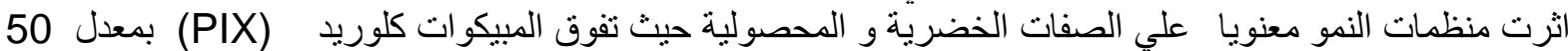

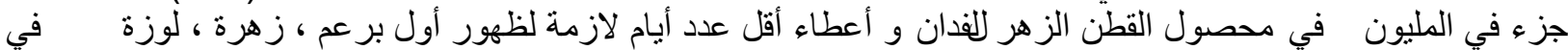
الموسمين وكذللك تفوق أيضا في دليل البذرة و نسبة التبكير بينما تفوق نفتالين أستيك أسيد NAA بمعدل 30 جزء 30 في في المليون

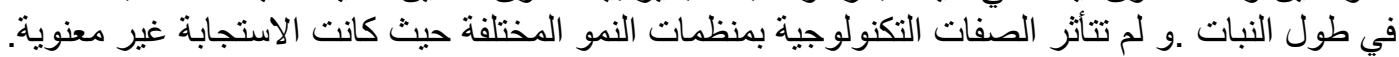

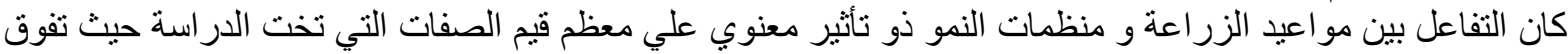

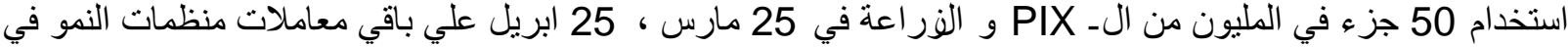

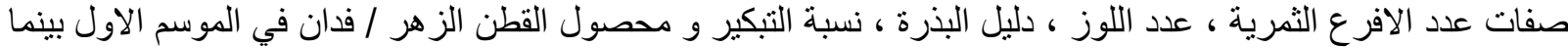

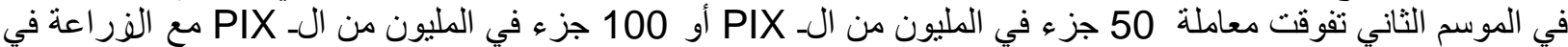

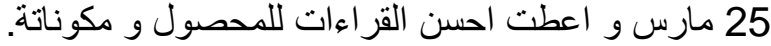

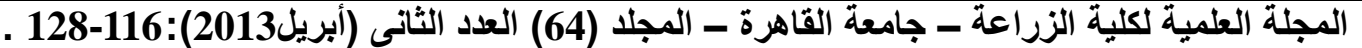

